\title{
Updates about Vertebrobasilar Insufficiency in Dizziness
}

\author{
Lilian Felipe* \\ Assistant Professor, Lamar University, USA \\ *Corresponding author: Lilian Felipe, Assistant Professor, Lamar University, USA
}

\begin{abstract}
Vertebrobasilar insufficiency (VBI) is a result of transitory ischemia of the vertebrobasilar arterial system (VBS) that can produce a variety of symptoms that are on their own are ambiguous. Symptoms include dizziness, vertigo, lightheadedness, headaches, visual changes, diplopia, ataxia, weakness in limbs, pain and stiffness of the neck. Vestibular and visual symptoms can arise suddenly and dissipate rapidly as well, all while preceding more serious symptoms like stroke and death. There are a variety of tests that audiologists and physical therapists can perform as screeners for this impairment, but imaging is an essential component of the diagnosis. Neuroimaging with angiography, magnetic resonance angiography (MRA), magnetic resonance imaging (MRI), and transracial Doppler tests are commonly used. Diagnosis and treating VBI requires a multidisciplinary and interdisciplinary approach. VBI has been documented to be exaggerated and over diagnosed in part due to the vague and transient nature of the symptomology. This paper will further outline the anatomy of the VBS, symptoms of VBI, risk factors, and diagnostic criteria.
\end{abstract}

Keywords: Atherosclerosis; Balance; Dizziness; Vertebrobasilar Arterial System; Vertebrobasilar Insufficiency

\section{Introduction}

The vertebrobasilar arterial system (VBS) supplies the vestibular and cochlear nuclei and is comprised of the vertebral artery (VA), basilar artery (BA), anterior-inferior cerebellar artery (AICA), and the posterior-inferior cerebellar artery (PICA) [1]. The VA and BA supply blood to the pons, medulla, cerebellum, mesencephalon, thalamus, occipital lobes, and of course, the peripheral and central vestibular system, as mentioned above [2]. The reason why changes in the VBS have a greater effect on the vestibular system rather than affecting both cochlear and vestibular function is that the cochlear system also received blood flow from the carotid artery which protects it from suffering the same impairments as the vestibular system which only received blood from the labyrinthine branches of the vertebrobasilar arteries [3]. Furthermore, the ischemia of the VBS can affect both peripheral and central vestibular structures by causing isolated dizziness attacks resulting from ischemia to the vestibular nuclei and/or vestibular cochlear nerve, or directly affecting the labyrinth [3]. The typical cause of hemodynamic changes in this system are the result of atherosclerosis; but other causes are embolism, and arterial dissection, or rarely migraine, fibromuscular dysplasia, and coagulopathies [3]. Considering the anatomy and physiology, it is easy to understand how changes or limitation in blood flow can induce disequilibrium, vertigo, and/or vision changes. Symptoms include dizziness, vertigo, nystagmus, imbalance, lightheadedness, headaches, mental confusion, aural fullness, tinnitus, hearing changes, nausea, vomiting, syncope, diplopia, blurred vision, blindness, ataxia, difficulty swallowing, dysarthria, pain and stiffness in neck or shoulder, and weakness of the extremities [1-4]. While it is possible for isolated and sudden attacks of vertigo to occur, vertigo alone is does not meet the diagnostic criteria for VBI [2,3]. Visual disturbances are commonly congruent with vertigo in VBI patients, but in fact, visual symptoms such as diplopia, visual hallucinations, changes in visual field, and blindness are more common than vertigo [3]. Patient report visual changes, diplopia, palsy of the oculomotor nerve, and seeing spots $[2,3]$. The onset of symptoms occurs rapidly and can be described as an attack by occurring during the change of position or suddenly reaching maximal affect within 5 minutes of the start and lasting anywhere from 2 minutes to 30 minutes commonly (but has been reported up to 24 hours) [4]. The resolution of the symptoms (or attack) occurs quickly. The frequency and antecedent of symptoms vary making it additionally difficult to diagnose based on patient complaints alone. The incidence is difficult to calculate as it has been recorded that VBI diagnosis has been exaggerated and inappropriately used to diagnose other conditions [5]. There is however a sex effect in that VBI is more likely to be found in men (rather than women) after the fourth decade of life [3]. 


\section{Discussion}

Differential diagnosis of VBI required careful examination to separate the symptoms from other conditions. Recording a detailed case history, performing the appropriate clinical measures, and having the essential imaging is required for adequate differential diagnosis. Case history will be necessary to identify possible risk factors such as: hyperlipidemia, hypertension, cerebrovascular diseases, carotid disease, heart disease, smoking, alcoholism, diabetes, and hyperglycemia [2,5]. Record and description of total symptoms at time of attack and clarifying any vagueness of response or other possible related incidences will be helpful. Also recognize that position changes like moving from laying/sitting to standing can induce symptoms. A vestibular assessment may be useful in differentiating the symptoms from other disorders. In the vestibular assessment the clinician may find that a patient with $\mathrm{VBI}$ and vestibular decruitment and hyperactive caloric responses $[2,4]$. Although hyperactive caloric responses may be observed, the two results of this test, canal paresis and directional preponderance, do not distinguish between an intracranial lesion or a labyrinthine impairment; however, the presence of decruitment/and or hyperactivity is indicative of an impairment as normal patients do not present with these clinical findings [4]. Some patients were also found to have abnormal function in the optokinetic pattern test, eye tracking, or visual suppression test in the videonystagmography (VNG) [1]. Another assessment conducted by the audiologist that may be useful in the Auditory Brainstem Response (ABR) when the ischemic is in the AICA displaying a substantial increase in interpeak latency between waves I-IV and II-IV [1].

A retrospective study done in China by Hu et al. [5] found that out of 773 patients diagnosed with VBI only 67 (8.67\%) of them had true VBI. Other conditions to consider are benign paroxysmal positional vertigo (BPPV), Meniere's disease, vestibular neuronitis, syncope, heart disease, abnormal blood pressure, sudden deafness, infectious diseases, and brain trauma [5]. Accordingly, neuroimaging will be useful in differential diagnosis. Tests include angiography, magnetic resonance angiography (MRA), magnetic resonance imaging (MRI) transracial Doppler, angio-tomography, and cardiologic studies $[3,4]$. Arteriography is highly useful in diagnosing VBI, but fewer patients are willing to have this test completed due to the risks of arterial catheters, low blood flow, or stroke [4]. The non-invasive test of MRA can be completed to identify possible occlusion or stenosis in neck or intracranial vessels $[1,3]$. This test has already been useful by identifying that the proximal regions of the vertebral arteries is the location of highest incidence causing VBI, as recorded with MRA [3]. When investigating the basilar artery, the MRA and angio-tomography are found to have similar sensitivity and specificity. The use of transcranial Doppler test is a low-cost, noninvasive, and pain-free test that is used to measure the speed and direction of intracranial arterial blood flow [3]. The plasticity index (PI) recording from the Doppler test is useful in predicting early hemodynamic intracranial variations; however, there is a sex effect in that speech and PI results are opposing for men and women as they age [3]. As evident with the above explanation, there are many useful tests that can be completed to aid in diagnosis of VBI and the need to multidisciplinary and interdisciplinary team is essential.

\section{Conclusion}

Understanding risk factors and possible symptoms is helpful in differential diagnosis, however, the risks and symptoms are broad and vague. Patients may present with the exact same symptomology but have varying diagnosis. The use of objective measures, such as caloric testing, and neuroimaging will be vital to accurately diagnosis. Due to the vast symptoms and fluctuating presentations, there is no set diagnostic criteria that can be applied to all patients. This is a concern to protect patients from the progression of the impairment which are stroke and possibly death. Correspondingly, more research needs to be conducted to understand the progression of stenosis as it gradually worsens over time.

\section{References}

1. Inui H, Yoneyama K, Kitaoku Y, Nakane M, Ohue S, et al. (1998) Four Cases of Vertebrobasilar Insufficiency. Acta Oto-Laryngologica 118(533): 4650 .

2. Asavasopon S, Jankoski J, Godges JJ (2005) Clinical diagnosis of vertebrobasial insufficiency: Resident's case problem. Journal of Orthopaedic \& Sports Physical Therapy 35(10): 645-650.

3. Lima Neto AC, Bittar R, Gattas GS, Bor Seng Shu E, Oliveira ML, et al. (2016) Pathophysiology and Diagnosis of Vertebrobasilar Insufficiency: A Review of the Literature. International archives of otorhinolaryngology 21(3): 302-307.

4. Kumar A, Mafee M, Dobben G, Whipple M, Pieri A (1998) Diagnosis of vertebrobasilar insufficiency: time to rethink established dogma? Ear, Nose, \& Throat Journal 77(12): 966-969, 972-974.

5. Xiaowei Hu, Qi Fang, Xia Yuan, Zhengchun Zhang, Wanli Dong (2012) Etiological diagnoses of vertebrobasilar insufficiency with dizziness in 773 patients over a 10 -year period in Suzhou, China. Pakistan Journal of Medical Sciences, 28(5): 860-864.

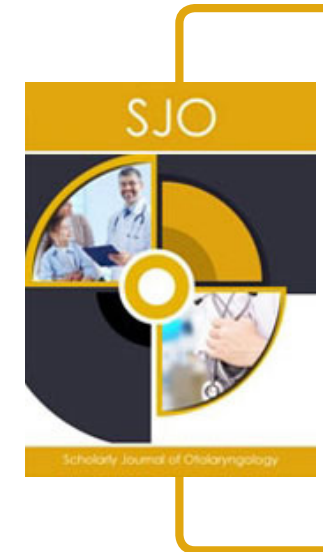

Scholarly Journal of Otolaryngology

\section{Assets of Publishing with us}

- Global archiving of articles

- Immediate, unrestricted online access

- Rigorous Peer Review Process

- Authors Retain Copyrights

- Unique DOI for all articles 